\title{
A SUZUKI FIXED POINT THEOREM FOR GENERALIZED MULTIVALUED MAPPINGS ON METRIC-LIKE SPACES
}

\author{
Hassen Aydi, Abdelbasset Felhi and Slah Sahmim
}

University of Dammam and King Faisal University, Saudi Arabia

\begin{abstract}
Based on a new papers of Aydi et al. in [7,8], where the concept of Hausdorff metric-like has been initiated, we introduce Suzuki type contractive multivalued mappings on metric-like spaces. We also establish several fixed point results involving such contractions. We show that many known fixed point results in literature are simple consequences of our theorems. Our obtained results are supported by some examples and an application.
\end{abstract}

\section{INTRODUCTION AND PRELIMINARIES}

The study of fixed points for multivalued contractions using the Hausdorff metric was initiated by Nadler ([17]). Since then, Aydi et al. in $[4,5]$ introduced the concept of a Hausdorff partial metric and provided some (common) fixed point results. Recently, Abbas et al. in [2] established some fixed point theorems for Suzuki type contractive multivalued mappings on partial Hausdorff metric spaces.

The purpose of this paper is to introduce the notion of Suzuki type contractive multivalued mappings on metric-like spaces via the notion of Hausdorff metric-like which a new concept introduced in recent papers of Aydi et al. $([7,8])$. We will establish some fixed point theorems involving such contractions on metric-like spaces.

Mention that metric-like spaces have been discovered by Amini-Harandi in [3] where some fixed point results have been established.

2010 Mathematics Subject Classification. 47H10, 54H25.

Key words and phrases. Hausdorff metric-like, multi-valued mapping, fixed point. 
Definition 1.1. Let $X$ be a nonempty set. A function $\sigma: X \times X \rightarrow[0, \infty)$ is said to be a metric-like (dislocated metric) on $X$ if for any $x, y, z \in X$, the following conditions hold:

$\left(\mathrm{P}_{1}\right) \sigma(x, y)=0 \Longrightarrow x=y$;

$\left(\mathrm{P}_{2}\right) \sigma(x, y)=\sigma(y, x)$

$\left(\mathrm{P}_{3}\right) \sigma(x, z) \leq \sigma(x, y)+\sigma(y, z)$.

The pair $(X, \sigma)$ is then called a metric-like (dislocated metric) space.

It is known that a partial metric $([16])$ is also a metric-like. So a trivial example of a metric-like space is the pair $([0, \infty), \sigma)$, where $\sigma:[0, \infty) \times$ $[0, \infty) \rightarrow[0, \infty)$ is defined as $\sigma(x, y)=\max \{x, y\}$.

In the following example, we give a metric-like which is neither a metric nor a partial metric.

Example 1.2. Let $X=\{0,1\}$ and $\sigma: X \times X \rightarrow[0, \infty)$ defined by

$$
\sigma(0,0)=2 \text { and } \sigma(x, y)=1 \text { if }(x, y) \neq(0,0) .
$$

Then, $(X, \sigma)$ is a metric-like space. Note that $\sigma$ is neither a metric since $\sigma(0,0) \neq 0$ nor a partial metric on $X$ because $\sigma(0,0)>\sigma(1,0)$.

Each metric-like $\sigma$ on $X$ generates a $T_{0}$ topology $\tau_{\sigma}$ on $X$ which has as a base the family open $\sigma$-balls $\left\{B_{\sigma}(x, \varepsilon): x \in X, \varepsilon>0\right\}$, where $B_{\sigma}(x, \varepsilon)=\{y \in$ $X:|\sigma(x, y)-\sigma(x, x)|<\varepsilon\}$, for all $x \in X$ and $\varepsilon>0$.

Observe that a sequence $\left\{x_{n}\right\}$ in a metric-like space $(X, \sigma)$ converges to a point $x \in X$, with respect to $\tau_{\sigma}$, if and only if $\sigma(x, x)=\lim _{n \rightarrow \infty} \sigma\left(x, x_{n}\right)$.

Definition 1.3. Let $(X, \sigma)$ be a metric-like space.

(a) A sequence $\left\{x_{n}\right\}$ in $X$ is said to be a Cauchy sequence if there exists $\lim _{n, m \rightarrow \infty} \sigma\left(x_{n}, x_{m}\right)$ and is finite.

(b) $(X, \sigma)$ is said to be complete if every Cauchy sequence $\left\{x_{n}\right\}$ in $X$ converges with respect to $\tau_{\sigma}$ to a point $x \in X$ such that $\lim _{n \rightarrow \infty} \sigma\left(x, x_{n}\right)=$ $\sigma(x, x)=\lim _{n, m \rightarrow \infty} \sigma\left(x_{n}, x_{m}\right)$.

We need in the sequel the following trivial inequality:

$$
\sigma(x, x) \leq 2 \sigma(x, y) \text { for all } x, y \in X .
$$

As in $[7,8]$, let $C B^{\sigma}(X)$ be the family of all nonempty, closed and bounded subsets of the metric-like space $(X, \sigma)$, induced by the metric-like $\sigma$. Note that the boundedness is given as follows: $A$ is a bounded subset in $(X, \sigma)$ if there exist $x_{0} \in X$ and $M \geq 0$ such that for all $a \in A$, we have $a \in B_{\sigma}\left(x_{0}, M\right)$, that is,

$$
\left|\sigma\left(x_{0}, a\right)-\sigma\left(x_{0}, x_{0}\right)\right|<M .
$$

The closedness is taken in $\left(X, \tau_{\sigma}\right)$ (where $\tau_{\sigma}$ is the topology induced by $\sigma$ ). Let $\bar{A}$ be the closure of $A$ with respect to the metric-like $\sigma$. We have 


\section{DEFINITION 1.4.}

$$
\begin{aligned}
a \in \bar{A} & \Longleftrightarrow B_{\sigma}(a, \epsilon) \cap A \neq \emptyset \quad \text { for all } \epsilon>0 \\
& \Longleftrightarrow \text { there exists }\left\{x_{n}\right\} \subset A, \quad x_{n} \rightarrow a \text { in }(X, \sigma) .
\end{aligned}
$$

If $A \in C B^{\sigma}(X)$, then $\bar{A}=A$.

For $A, B \in C B^{\sigma}(X)$ and $x \in X$, define

$\sigma(x, A)=\inf \{\sigma(x, a), a \in A\}, \delta_{\sigma}(A, B)=\sup \{\sigma(a, B): a \in A\} \quad$ and

$\delta_{\sigma}(B, A)=\sup \{\sigma(b, A): b \in B\}$.

Lemma $1.5([7,8])$. Let $(X, \sigma)$ be a metric-like space and $A$ be any nonempty set in $(X, \sigma)$, then

$$
\text { if } \sigma(a, A)=0, \quad \text { then } a \in \bar{A} .
$$

Let $(X, \sigma)$ be a metric-like space. For $A, B \in C B^{\sigma}(X)$, define

$$
H_{\sigma}(A, B)=\max \left\{\delta_{\sigma}(A, B), \delta_{\sigma}(B, A)\right\} .
$$

We also have some properties of $H_{\sigma}: C B^{\sigma}(X) \times C B^{\sigma}(X) \rightarrow[0, \infty)$.

Proposition $1.6([7,8])$. Let $(X, \sigma)$ be a metric-like space. For any $A, B, C \in C B^{\sigma}(X)$, we have the following:

$$
\begin{aligned}
&(i) \quad: \quad H_{\sigma}(A, A)=\delta_{\sigma}(A, A)=\sup \{\sigma(a, A): a \in A\} ; \\
&(i i): H_{\sigma}(A, B)=H_{\sigma}(B, A) ; \\
&(\text { iii }): H_{\sigma}(A, B)=H_{\sigma}(B, A)=0 \text { implies that } A=B ; \\
&(\text { iv }): H_{\sigma}(A, B) \leq H_{\sigma}(A, C)+H_{\sigma}(C, B) .
\end{aligned}
$$

In view of Proposition 1.6, the mapping $H_{\sigma}: C B^{\sigma}(X) \times C B^{\sigma}(X) \rightarrow$ $[0,+\infty)$ is called a Hausdorff metric-like induced by $\sigma$.

From now on, we denote by

$$
M(x, y):=\max \left\{\sigma(x, y), \sigma(x, T x), \sigma(y, T y), \frac{1}{4}\{\sigma(x, T y)+\sigma(y, T x)\}\right\}
$$

for a multivalued map $T: X \rightarrow C B^{\sigma}(X)$ and $x, y \in X$.

Also, let $\psi:[0,1) \rightarrow(0,1]$ the non-increasing function defined by

$$
\psi(r)=\left\{\begin{array}{l}
1, \quad 0 \leq r<\frac{1}{2}, \\
1-r, \quad \frac{1}{2} \leq r<1 .
\end{array}\right.
$$

In this paper, we introduce the concept of Suzuki type contractive multivalued mappings on metric-like spaces. We establish some fixed point results for multivalued mappings involving above contractions. We will present some examples and an application. 


\section{FIXED POINT OF MULTI-VALUED CONTRACTION MAPPINGS}

We start with the following useful lemmas.

Lemma 2.1. Let $A, B \in C B^{\sigma}(X)$ and $a \in A$. Then, for all $\epsilon>0$, there exists a point $b \in B$ such that $\sigma(a, b) \leq H_{\sigma}(A, B)+\epsilon$.

Lemma 2.2. Let $A, B \in C B^{\sigma}(X)$ and $a \in A$. Suppose that $\sigma(a, B)>0$. Then, for each $0 \leq h<1$, there exists $b \in B$ such that $h \sigma(a, b)<\sigma(a, B)$.

Proof. We argue by contradiction, that is, there exists $0 \leq h<1$, such that for all $b \in B$, there is $h \sigma(a, b) \geq \sigma(a, B)$. Then, $h \sigma(a, B) \geq \sigma(a, B)$. Hence, $h \geq 1$, which is a contradiction.

Lemma 2.3. Let $A, B, C \in C B^{\sigma}(X)$ and $a \in A$. Then

$$
\sigma(a, B) \leq \sigma(a, C)+H_{\sigma}(C, B) .
$$

Now, we state and prove our main result.

THEOREM 2.4. Let $(X, \sigma)$ be a complete metric-like space and $T: X \rightarrow$ $C B^{\sigma}(X)$ be a multivalued mapping. Suppose there exists $0 \leq r<1$ such that $T$ satisfies the condition

$$
\psi(r) \sigma(x, T x) \leq \sigma(x, y) \quad \text { implies } \quad H_{\sigma}(T x, T y) \leq r M(x, y)
$$

for all $x, y \in X$, where $\psi$ is defined by (1.3). Then, $T$ has a fixed point, that is, there exists a point $x^{\star} \in X$ such that $x^{\star} \in T x^{\star}$.

Proof. Let $x_{0} \in X$ and $x_{1} \in T x_{0}$. Clearly, if $\sigma\left(x_{0}, x_{1}\right)=0$, then $x_{0}=x_{1}$ and $x_{0}$ is a fixed point of $T$. Assume that $\sigma\left(x_{0}, x_{1}\right)>0$. Since $T x_{0}, T x_{1} \in$ $C B^{\sigma}(X)$ and $x_{1} \in T x_{0}$, so by Lemma 2.1, there exists a point $x_{2} \in T x_{1}$ such that

$$
\sigma\left(x_{1}, x_{2}\right) \leq H_{\sigma}\left(T x_{0}, T x_{1}\right)+\frac{1-r}{2} M\left(x_{1}, x_{0}\right) .
$$

But $\psi(r) \leq 1$, so

$$
\psi(r) \sigma\left(x_{0}, T x_{0}\right) \leq \sigma\left(x_{0}, T x_{0}\right) \leq \sigma\left(x_{0}, x_{1}\right) .
$$

Thus, from $(2.1)$

$$
\begin{aligned}
\sigma\left(x_{1}, x_{2}\right) & \leq H_{\sigma}\left(T x_{0}, T x_{1}\right)+\frac{1-r}{2} M\left(x_{0}, x_{1}\right) \\
& \leq r M\left(x_{0}, x_{1}\right)+\frac{1-r}{2} M\left(x_{1}, x_{0}\right) \\
& =\frac{1+r}{2} M\left(x_{0}, x_{1}\right) .
\end{aligned}
$$

If $\sigma\left(x_{2}, x_{1}\right)=0$, then $x_{2}=x_{1}$ and so $x_{1}$ is a fixed point of $T$. Assume that $\sigma\left(x_{2}, x_{1}\right)>0$. Then, by Lemma 2.1 , there exists a point $x_{3} \in T x_{2}$ such that

$$
\sigma\left(x_{3}, x_{2}\right) \leq H_{\sigma}\left(T x_{2}, T x_{1}\right)+\frac{1-r}{2} M\left(x_{2}, x_{1}\right)
$$


Again $\psi(r) \leq 1$, so

$$
\psi(r) \sigma\left(x_{1}, T x_{1}\right) \leq \sigma\left(x_{1}, T x_{1}\right) \leq \sigma\left(x_{1}, x_{2}\right) .
$$

From (2.1), we have

$$
\begin{aligned}
\sigma\left(x_{2}, x_{3}\right) & \leq H_{\sigma}\left(T x_{1}, T x_{2}\right)+\frac{1-r}{2} M\left(x_{1}, x_{2}\right) \\
& \leq r M\left(x_{1}, x_{2}\right)+\frac{1-r}{2} M\left(x_{1}, x_{2}\right) \\
& =\frac{1+r}{2} M\left(x_{1}, x_{2}\right) .
\end{aligned}
$$

Continuing in this fashion, we produce a sequence $\left\{x_{n}\right\} \subset X$ such that $x_{n+1} \in$ $T x_{n}$ and $\sigma\left(x_{n}, x_{n+1}\right)>0$ with

$$
\sigma\left(x_{n}, x_{n+1}\right) \leq H_{\sigma}\left(T x_{n-1}, T x_{n}\right)+\frac{1-r}{2} M\left(x_{n-1}, x_{n}\right) .
$$

We have

$$
\psi(r) \sigma\left(x_{n-1}, T x_{n-1}\right) \leq \sigma\left(x_{n-1}, T x_{n-1}\right) \leq \sigma\left(x_{n-1}, x_{n}\right) .
$$

From (2.1)

$$
\begin{aligned}
0<\sigma\left(x_{n}, x_{n+1}\right) & \leq H_{\sigma}\left(T x_{n-1}, T x_{n}\right)+\frac{1-r}{2} M\left(x_{n-1}, x_{n}\right) \\
& \leq r M\left(x_{n-1}, x_{n}\right)+\frac{1-r}{2} M\left(x_{n-1}, x_{n}\right) \\
& =\frac{1+r}{2} M\left(x_{n-1}, x_{n}\right),
\end{aligned}
$$

where

$$
\begin{aligned}
M\left(x_{n-1}, x_{n}\right)= & \max \left\{\sigma\left(x_{n-1}, x_{n}\right), \sigma\left(x_{n-1}, T x_{n-1}\right), \sigma\left(x_{n}, T x_{n}\right),\right. \\
& \left.\frac{1}{4}\left[\sigma\left(x_{n-1}, T x_{n}\right)+\sigma\left(x_{n}, T x_{n-1}\right)\right]\right\} \\
\leq & \max \left\{\sigma\left(x_{n-1}, x_{n}\right), \sigma\left(x_{n-1}, x_{n}\right), \sigma\left(x_{n}, x_{n+1}\right),\right. \\
& \left.\frac{1}{4}\left[\sigma\left(x_{n-1}, x_{n+1}\right)+\sigma\left(x_{n}, x_{n}\right)\right]\right\} \\
\leq & \max \left\{\sigma\left(x_{n-1}, x_{n}\right), \sigma\left(x_{n}, x_{n+1}\right), \frac{1}{4}\left[3 \sigma\left(x_{n-1}, x_{n}\right)+\sigma\left(x_{n}, x_{n+1}\right)\right]\right\} \\
= & \max \left\{\sigma\left(x_{n-1}, x_{n}\right), \sigma\left(x_{n}, x_{n+1}\right)\right\} .
\end{aligned}
$$

Thus, for all $n \in \mathbb{N}$

$$
0<\sigma\left(x_{n}, x_{n+1}\right) \leq \frac{1+r}{2} \max \left\{\sigma\left(x_{n-1}, x_{n}\right), \sigma\left(x_{n}, x_{n+1}\right)\right\} .
$$

If $\sigma\left(x_{n}, x_{n+1}\right)>\sigma\left(x_{n-1}, x_{n}\right)$ for some $n$, so due to the fact that $0 \leq r<1$,

$$
0<\sigma\left(x_{n}, x_{n+1}\right) \leq \frac{1+r}{2} \sigma\left(x_{n}, x_{n+1}\right)<\sigma\left(x_{n}, x_{n+1}\right)
$$


which is a contradiction. Then, for all $n \in \mathbb{N}$

$$
\sigma\left(x_{n}, x_{n+1}\right) \leq \frac{1+r}{2} \sigma\left(x_{n-1}, x_{n}\right) .
$$

Moreover, by induction

$$
\sigma\left(x_{n}, x_{n+1}\right) \leq\left(\frac{1+r}{2}\right)^{n} \sigma\left(x_{0}, x_{1}\right), \quad \text { for all } n=0,1, \ldots
$$

Since $r \in[0,1)$, then $\sum_{n}\left(\frac{1+r}{2}\right)^{n}<\infty$. So, for all $p \in \mathbb{N}$

$$
\begin{aligned}
\sigma\left(x_{n}, x_{n+p}\right) & \leq \sum_{k=n}^{n+p-1} \sigma\left(x_{k}, x_{k+1}\right) \leq \sum_{k=n}^{n+p-1}\left(\frac{1+r}{2}\right)^{k} \sigma\left(x_{0}, x_{1}\right) \\
& \leq \sum_{k=n}^{\infty}\left(\frac{1+r}{2}\right)^{k} \sigma\left(x_{0}, x_{1}\right) \rightarrow 0, \text { as } n \rightarrow \infty .
\end{aligned}
$$

Thus, by symmetry of $\sigma$

$$
\lim _{n, m \rightarrow \infty} \sigma\left(x_{n}, x_{m}\right)=0 .
$$

This yields that the sequence $\left\{x_{n}\right\}$ is a $\sigma$-Cauchy. By completeness of $(X, \sigma)$, there exists $x^{\star} \in X$ such that

$$
\lim _{n \rightarrow \infty} \sigma\left(x_{n}, x^{\star}\right)=\sigma\left(x^{\star}, x^{\star}\right)=\lim _{n, m \rightarrow \infty} \sigma\left(x_{n}, x_{m}\right)=0 .
$$

We will show that

$$
\sigma\left(x^{\star}, T x\right) \leq r \max \left\{\sigma\left(x^{\star}, x\right), \sigma(x, T x)\right\} \text { for all } x \neq x^{\star} .
$$

First, take $x \neq x^{\star} \in X$ and so $\sigma\left(x, x^{\star}\right)>0$. Since $\lim _{n \rightarrow \infty} \sigma\left(x_{n}, x^{\star}\right)=0$, then there exists $N \in \mathbb{N}$ such that

$$
\sigma\left(x_{n}, x^{\star}\right) \leq \frac{1}{3} \sigma\left(x, x^{\star}\right), \quad \text { for all } n \geq N .
$$

Then, we have

$$
\begin{aligned}
\psi(r) \sigma\left(x_{n}, T x_{n}\right) & \leq \sigma\left(x_{n}, T x_{n}\right) \leq \sigma\left(x_{n}, x_{n+1}\right) \\
& \leq \sigma\left(x_{n}, x^{\star}\right)+\sigma\left(x^{\star}, x_{n+1}\right) \leq \frac{2}{3} \sigma\left(x, x^{\star}\right) .
\end{aligned}
$$

By (2.5) and the triangular inequality, we can write

$$
\frac{2}{3} \sigma\left(x, x^{\star}\right) \leq \sigma\left(x, x^{\star}\right)-\sigma\left(x_{n}, x^{\star}\right) \leq \sigma\left(x, x_{n}\right), \quad \text { for all } n \geq N .
$$

Combining (2.6) and (2.7), we get

$$
\psi(r) \sigma\left(x_{n}, T x_{n}\right) \leq \sigma\left(x, x_{n}\right), \quad \text { for all } n \geq N .
$$

Therefore, from (2.1)

$$
H_{\sigma}\left(T x, T x_{n}\right) \leq r M\left(x, x_{n}\right), \quad \text { for all } n \geq N,
$$


where

$$
M\left(x, x_{n}\right)=\max \left\{\sigma\left(x, x_{n}\right), \sigma(x, T x), \sigma\left(x_{n}, T x_{n}\right), \frac{1}{4}\left[\sigma\left(x, T x_{n}\right)+\sigma\left(x_{n}, T x\right)\right]\right\} .
$$

Note that

$M\left(x, x_{n}\right) \leq \max \left\{\sigma\left(x, x_{n}\right), \sigma(x, T x), \sigma\left(x_{n}, x_{n+1}\right), \frac{1}{4}\left[\sigma\left(x, x_{n+1}\right)+\sigma\left(x_{n}, T x\right)\right]\right\}$.

We obtain

$$
\begin{aligned}
& \sigma\left(x^{\star}, T x\right) \leq \sigma\left(x^{\star}, x_{n+1}\right)+\sigma\left(x_{n+1}, T x\right) \\
& \leq \sigma\left(x^{\star}, x_{n+1}\right)+H_{\sigma}\left(T x_{n}, T x\right) \\
& \leq \sigma\left(x^{\star}, x_{n+1}\right)+r \max \left\{\sigma\left(x, x_{n}\right), \sigma(x, T x), \sigma\left(x_{n}, T x_{n}\right),\right. \\
&\left.\frac{1}{4}\left[\sigma\left(x, T x_{n}\right)+\sigma\left(x_{n}, T x\right)\right]\right\} \\
& \leq \sigma\left(x^{\star}, x_{n+1}\right)+r \max \left\{\sigma\left(x, x_{n}\right), \sigma(x, T x), \sigma\left(x_{n}, x_{n+1}\right),\right. \\
&\left.\frac{1}{4}\left[\sigma\left(x, x_{n+1}\right)+\sigma\left(x_{n}, x\right)+\sigma(x, T x)\right]\right\} .
\end{aligned}
$$

In view of $(2.3)$

$$
\lim _{n \rightarrow \infty} \sigma\left(x, x_{n}\right)=\sigma\left(x, x^{\star}\right), \quad \lim _{n \rightarrow \infty} \sigma\left(x_{n}, x_{n+1}\right)=0 .
$$

Letting $n \rightarrow \infty$, we get

$$
\begin{aligned}
\sigma\left(x^{\star}, T x\right) & \leq r \max \left\{\sigma\left(x, x^{\star}\right), \sigma(x, T x), \frac{1}{4}\left[2 \sigma\left(x, x^{\star}\right)+\sigma(x, T x)\right]\right\} \\
& \leq r \max \left\{\sigma\left(x, x^{\star}\right), \sigma(x, T x)\right\} .
\end{aligned}
$$

This completes the proof of (2.4). Now, we will show that $x^{\star} \in T x^{\star}$. First, consider the case $0 \leq r<\frac{1}{2}$. Suppose, on the contrary, that $x^{\star} \notin T x^{\star}$. By Lemma 2.2, there exists $a \in T x^{\star}$ such that

$$
2 r \sigma\left(a, x^{\star}\right)<\sigma\left(x^{\star}, T x^{\star}\right) .
$$

Since $a \in T x^{\star}$, then $a \neq x^{\star}$. From (2.4), we have

$$
\sigma\left(x^{\star}, T a\right) \leq r \max \left\{\sigma\left(x^{\star}, a\right), \sigma(a, T a)\right\} .
$$

We have $\psi(r) \sigma\left(x^{\star}, T x^{\star}\right) \leq \sigma\left(x^{\star}, T x^{\star}\right) \leq \sigma\left(x^{\star}, a\right)$. Then, from (2.1)

$H_{\sigma}\left(T x^{\star}, T a\right) \leq r \max \left\{\sigma\left(x^{\star}, a\right), \sigma\left(x^{\star}, T x^{\star}\right), \sigma(a, T a), \frac{1}{4}\left[\sigma\left(a, T x^{\star}\right)+\sigma\left(x^{\star}, T a\right)\right]\right\}$.

By triangle inequality

$$
\begin{aligned}
\sigma\left(a, T x^{\star}\right)+\sigma\left(x^{\star}, T a\right) & \leq 2 \sigma\left(a, x^{\star}\right)+\sigma\left(x^{\star}, T x^{\star}\right)+\sigma(a, T a) \\
& \leq 4 \max \left\{\sigma\left(x^{\star}, a\right), \sigma\left(x^{\star}, T x^{\star}\right), \sigma(a, T a)\right\} .
\end{aligned}
$$

Then

$$
H_{\sigma}\left(T x^{\star}, T a\right) \leq r \max \left\{\sigma\left(x^{\star}, a\right), \sigma\left(x^{\star}, T x^{\star}\right), \sigma(a, T a)\right\} .
$$


Again, having $\sigma\left(x^{\star}, T x^{\star}\right) \leq \sigma\left(x^{\star}, a\right)$, so

$$
\sigma(a, T a) \leq H_{\sigma}\left(T x^{\star}, T a\right) \leq r \max \left\{\sigma\left(x^{\star}, a\right), \sigma(a, T a)\right\} .
$$

If $\sigma(a, T a)=0$, then by Lemma 1.5, $a \in \overline{T a}$, and then $a$ is a fixed point of $T$.

Suppose that $\sigma(a, T a)>0$. So if $\sigma(a, T a)>\sigma\left(a, x^{\star}\right)$, then

$$
\sigma(a, T a) \leq r \sigma(a, T a)<\sigma(a, T a)
$$

which is a contradiction. Then

$$
\sigma(a, T a) \leq r \sigma\left(x^{\star}, a\right)<\sigma\left(x^{\star}, a\right) .
$$

Therefore, by Lemma 2.3 and using (2.4) and (2.9)

$$
\begin{aligned}
\sigma\left(x^{\star}, T x^{\star}\right) & \leq \sigma\left(x^{\star}, T a\right)+H_{\sigma}\left(T a, T x^{\star}\right) \\
& \leq \sigma\left(x^{\star}, T a\right)+r \max \left\{\sigma\left(x^{\star}, a\right), \sigma(a, T a)\right\} \\
& \leq 2 r \max \left\{\sigma\left(x^{\star}, a\right), \sigma(a, T a)\right\} \leq 2 r \sigma\left(x^{\star}, a\right)
\end{aligned}
$$

which is a contradiction. Thus, $x^{\star} \in T x^{\star}$.

Now, we consider the case $\frac{1}{2} \leq r<1$. First, we will prove that

$$
\begin{array}{r}
H_{\sigma}\left(T x, T x^{\star}\right) \leq r \max \left\{\sigma\left(x, x^{\star}\right), \sigma(x, T x), \sigma\left(x^{\star}, T x^{\star}\right),\right. \\
\left.\frac{1}{4}\left[\sigma\left(x, T x^{\star}\right)+\sigma\left(x^{\star}, T x\right)\right]\right\},
\end{array}
$$

for all $x \neq x^{\star}$.

Let us take $x \in X$ such that $\sigma\left(x, x^{\star}\right)>0$. Then, by definition of the infimum, there exists $y_{n} \in T x$ such that for each $n \in \mathbb{N}$,

$$
\sigma\left(x^{\star}, y_{n}\right) \leq \sigma\left(x^{\star}, T x\right)+\frac{1}{n} \sigma\left(x, x^{\star}\right) .
$$

By triangular inequality and using (2.4)

$$
\begin{aligned}
\sigma(x, T x) & \leq \sigma\left(x, y_{n}\right) \leq \sigma\left(x, x^{\star}\right)+\sigma\left(x^{\star}, y_{n}\right) \\
& \leq \sigma\left(x, x^{\star}\right)+\sigma\left(x^{\star}, T x\right)+\frac{1}{n} \sigma\left(x, x^{\star}\right) \\
& \leq\left(1+\frac{1}{n}\right) \sigma\left(x, x^{\star}\right)+r \max \left\{\sigma\left(x^{\star}, x\right), \sigma(x, T x)\right\} .
\end{aligned}
$$

CASE 1: If $\sigma\left(x, x^{\star}\right) \geq \sigma(x, T x)$, then

$$
\sigma(x, T x) \leq\left(1+r+\frac{1}{n}\right) \sigma\left(x, x^{\star}\right)
$$

Letting $n \rightarrow \infty$

$$
\sigma(x, T x) \leq(1+r) \sigma\left(x, x^{\star}\right) .
$$

By definition of $\psi$ in the case $\frac{1}{2} \leq r<1$, we have

$$
\psi(r) \sigma(x, T x)=(1-r) \sigma(x, T x) \leq\left(1-r^{2}\right) \sigma\left(x, x^{\star}\right) \leq \sigma\left(x, x^{\star}\right) .
$$


Then, from $(2.1)$

$$
\begin{gathered}
H_{\sigma}\left(T x, T x^{\star}\right) \leq r \max \left\{\sigma\left(x, x^{\star}\right), \sigma(x, T x), \sigma\left(x^{\star}, T x^{\star}\right),\right. \\
\left.\frac{1}{4}\left[\sigma\left(x, T x^{\star}\right)+\sigma\left(x^{\star}, T x\right)\right]\right\} .
\end{gathered}
$$

CASE 2: If $\sigma\left(x, x^{\star}\right)<\sigma(x, T x)$, we get

$$
\sigma(x, T x) \leq\left(1+\frac{1}{n}\right) \sigma\left(x, x^{\star}\right)+r \sigma(x, T x) .
$$

Thus

$$
(1-r) \sigma(x, T x) \leq\left(1+\frac{1}{n}\right) \sigma\left(x, x^{\star}\right) .
$$

We pass to the limit when $n \rightarrow \infty$

$$
\psi(r) \sigma(x, T x) \leq \sigma\left(x, x^{\star}\right) .
$$

By (2.1), we obtain

$$
\begin{gathered}
H_{\sigma}\left(T x, T x^{\star}\right) \leq r \max \left\{\sigma\left(x, x^{\star}\right), \sigma(x, T x), \sigma\left(x^{\star}, T x^{\star}\right),\right. \\
\left.\frac{1}{4}\left[\sigma\left(x, T x^{\star}\right)+\sigma\left(x^{\star}, T x\right)\right]\right\} .
\end{gathered}
$$

This ends the proof of (2.10) in the two cases.

Since $x_{n} \neq x_{n+1}$ for all $n \in \mathbb{N}$, then $x_{n} \neq x^{\star}$ or $x_{n+1} \neq x^{\star}$ for all $n \in \mathbb{N}$. So, we assume that $x_{n} \neq x^{\star}$ for infinitely many $n$. Thus, we may suppose that $x_{n} \neq x^{\star}$ for all $n \in \mathbb{N}$.

Suppose that $\sigma\left(x^{\star}, T x^{\star}\right)>0$. We have for all $n \in \mathbb{N}$

$$
\sigma\left(x^{\star}, T x^{\star}\right) \leq \sigma\left(x^{\star}, x_{n+1}\right)+\sigma\left(x_{n+1}, T x^{\star}\right) .
$$

Since $x_{n+1} \in T x_{n}$, it follows

$$
\sigma\left(x^{\star}, T x^{\star}\right) \leq \sigma\left(x^{\star}, x_{n+1}\right)+H_{\sigma}\left(T x_{n}, T x^{\star}\right) .
$$

Then, by (2.10), we obtain

$$
\begin{gathered}
\sigma\left(x^{\star}, T x^{\star}\right) \leq \sigma\left(x^{\star}, x_{n+1}\right)+r \max \left\{\sigma\left(x_{n}, x^{\star}\right), \sigma\left(x_{n}, T x_{n}\right), \sigma\left(x^{\star}, T x^{\star}\right),\right. \\
\left.\frac{1}{4}\left[\sigma\left(x_{n}, T x^{\star}\right)+\sigma\left(x^{\star}, T x_{n}\right)\right]\right\} .
\end{gathered}
$$

We know that

$$
\begin{aligned}
& \sigma\left(x_{n}, T x_{n}\right) \leq \sigma\left(x_{n}, x_{n+1}\right), \\
& \sigma\left(x_{n}, T x^{\star}\right) \leq \sigma\left(x_{n}, x^{\star}\right)+\sigma\left(x^{\star}, T x^{\star}\right), \\
& \sigma\left(x^{\star}, T x_{n}\right) \leq \sigma\left(x^{\star}, x_{n+1}\right) .
\end{aligned}
$$

Then, by taking $n \rightarrow \infty$, we get

$$
0<\sigma\left(x^{\star}, T x^{\star}\right) \leq r \sigma\left(x^{\star}, T x^{\star}\right)<\sigma\left(x^{\star}, T x^{\star}\right)
$$

which is a contradiction. Hence, $\sigma\left(x^{\star}, T x^{\star}\right)=0$ and so $x^{\star} \in \overline{T x^{\star}}=T x^{\star}$. Then, $x^{\star}$ is a fixed point of $T$. 
2.1. Some consequences. We state the following simple corollaries as consequences of Theorem 2.4 .

Corollary 2.5. Let $(X, \sigma)$ be a complete metric-like space and $T: X \rightarrow$ $C B^{\sigma}(X)$ be a multivalued mapping. Suppose that there exists $0 \leq r<1$ such that $T$ satisfies the condition

$$
\begin{gathered}
\psi(r) \sigma(x, T x) \leq \sigma(x, y) \\
\text { implies } \\
H_{\sigma}(T x, T y) \leq r \max \{\sigma(x, y), \sigma(x, T x), \sigma(y, T y)\}
\end{gathered}
$$

for all $x, y \in X$, where $\psi$ is defined by (1.3). Then, $T$ has a fixed point in $X$.

Proof. It suffices to consider Theorem 2.4 and to use the fact that (2.11) implies (2.1).

We also have the following simple corollaries.

Corollary 2.6. Let $(X, \sigma)$ be a complete metric-like space and $T: X \rightarrow$ $C B^{\sigma}(X)$ be a multivalued mapping. Suppose there exists $0 \leq r<1$ such that $T$ satisfies the condition

$$
\begin{gathered}
\psi(r) \sigma(x, T x) \leq \sigma(x, y) \\
\text { implies } \\
H_{\sigma}(T x, T y) \leq \frac{r}{3}\{\sigma(x, y)+\sigma(x, T x)+\sigma(y, T y)\}
\end{gathered}
$$

for all $x, y \in X$, where $\psi$ is defined by (1.3). Then, $T$ has a fixed point in $X$.

Corollary 2.7. Let $(X, \sigma)$ be a complete metric-like space and $T: X \rightarrow$ $X$ be a mapping. Suppose there exists $0 \leq r<1$ such that $T$ satisfies the condition

$$
\psi(r) \sigma(x, T x) \leq \sigma(x, y) \quad \text { implies } \quad \sigma(T x, T y) \leq r M(x, y)
$$

for all $x, y \in X$, where $\psi$ is defined by (1.3). Then, $T$ has a fixed point, that is, there exists a point $x^{\star} \in X$ such that $x^{\star}=T x^{\star}$.

\section{EXAMPLES AND APPLICATION}

3.1. Examples. First, we give the following illustrative examples.

ExAmple 3.1. Let $X=\{0,1,2\}$ and $\sigma: X \times X \rightarrow \mathbb{R}+$ defined by

$$
\begin{aligned}
& \sigma(0,0)=\sigma(1,1)=0, \quad \sigma(2,2)=\frac{23}{48} \\
& \sigma(0,1)=\sigma(1,0)=\frac{1}{3}, \quad \sigma(0,2)=\sigma(2,0)=\frac{11}{24}, \quad \sigma(1,2)=\sigma(2,1)=\frac{1}{2} .
\end{aligned}
$$

Then $(X, \sigma)$ is a complete metric-like space. Note that $\sigma$ is not a partial metric on $X$ because $\sigma(2,2)>\sigma(2,0)$. 
Define the map $T: X \rightarrow C B^{\sigma}(X)$ by

$$
T x= \begin{cases}\{0\}, & x \neq 2 \\ \{0,1\}, & x=2 .\end{cases}
$$

Therefore, we get

$$
\begin{aligned}
& \max \{\sigma(x, T x): x \in X\}=\max \left\{0, \frac{1}{3}, \frac{11}{24}\right\}=\frac{11}{24}, \\
& \min \{\sigma(x, y): x, y \in X \text { and } x \neq y\}=\frac{1}{3} .
\end{aligned}
$$

Choose $r=\frac{3}{4}$ and $\psi(r)=\frac{1}{4}$. Then

$$
\begin{aligned}
\psi(r) \max \{\sigma(x, T x): x \in X\} & =\frac{1}{4} \times \frac{11}{24}=\frac{11}{96} \\
& <\frac{1}{3}=\min \{\sigma(x, y): x, y \in X \text { and } x \neq y\} .
\end{aligned}
$$

Thus

$$
\psi(r) \sigma(x, T x): x \in X\} \leq \sigma(x, y)
$$

for all $x, y \in X$ with $x \neq y$. Consequently, we show

$$
H_{\sigma}(T x, T y) \leq r M(x, y),
$$

for all $x, y \in X$. For this, we distinguish the following cases:

case 1: $x, y \in\{0,1\}$. We have

$$
H_{\sigma}(T x, T y)=\sigma(0,0)=0 \leq r \sigma(x, y) \leq r M(x, y) .
$$

case 2: $x \in\{0,1\}, y=2$. We have

$$
\begin{aligned}
H_{\sigma}(T x, T y)=H_{\sigma}(\{0\},\{0,1\}) & =\max \{\sigma(0,\{0,1\}), \max \{\sigma(0,0), \sigma(0,1)\}\} \\
& =\max \left\{\min \{\sigma(0,0), \sigma(0,1)\}, \frac{1}{3}\right\} \\
& =\frac{1}{3} \leq r \sigma(x, y) \leq r M(x, y) .
\end{aligned}
$$

case 3: $x=y=2$. We have

$$
\begin{aligned}
H_{\sigma}(T x, T y)=H_{\sigma}(\{0,1\},\{0,1\}) & =\max \{\sigma(0,\{0,1\}), \sigma(1,\{0,1\})\} \\
& =\min \{\sigma(0,1), \sigma(1,1)\}=0 \\
& \leq \operatorname{ro}(2,2) \leq r M(2,2) .
\end{aligned}
$$

Hence, for all $x, y \in X$

$$
\left.\psi(r) \sigma(x, T x) \leq \sigma(x, y) \text { implies } H_{\sigma}(T x, T y)\right) \leq r M(x, y) .
$$

Thus, all hypotheses of Theorem 2.4 are satisfied. Then, $T$ has a fixed point which is $u=0$. 
Example 3.2. Let $X=\{0,1,2\}$ and $\sigma: X \times X \rightarrow \mathbb{R}+$ be defined by $\sigma(0,0)=1, \quad \sigma(1,1)=3, \quad \sigma(2,2)=0$ $\sigma(0,1)=\sigma(1,0)=4, \quad \sigma(0,2)=\sigma(2,0)=3, \quad \sigma(1,2)=\sigma(2,1)=7$.

Then, $(X, \sigma)$ is a complete metric-like space. Note that $\sigma$ is not a partial metric on $X$ since $\sigma(2,1)>\sigma(2,0)+\sigma(0,1)-\sigma(0,0)$. Define the map $T$ : $X \rightarrow C B^{\sigma}(X)$ by

$$
T x=\left\{\begin{array}{l}
\{2\}, \quad x \neq 1 \\
\{0,2\}, \quad x=1 .
\end{array}\right.
$$

Therefore, we get

$$
\max \{\sigma(x, T x): x \in X\}=4, \quad \min \{\sigma(x, y): x, y \in X \text { and } x \neq y\}=3 .
$$

Choose $r=\frac{4}{5}$ and $\psi(r)=\frac{1}{5}$. Then

$$
\psi(r) \max \{\sigma(x, T x): x \in X\}=\frac{4}{5}<3=\min \{\sigma(x, y): x, y \in X \text { and } x \neq y\} .
$$

Thus

$$
\psi(r) \sigma(x, T x): x \in X\} \leq \sigma(x, y)
$$

for all $x, y \in X$ with $x \neq y$. Consequently, we show that

$$
H_{\sigma}(T x, T y) \leq r M(x, y)
$$

for all $x, y \in X$. For this, we consider the following cases: case(1): $x, y \in\{0,2\}$. We have

$$
H_{\sigma}(T x, T y)=\sigma(2,2)=0 \leq r M(x, y) .
$$

case(2): $x \in\{0,2\}, y=1$. We have

$$
\begin{aligned}
H_{\sigma}(T x, T y)=H_{\sigma}(\{2\},\{0,2\}) & =\max \{\sigma(2,\{0,2\}), \max \{\sigma(2,2), \sigma(0,2)\}\} \\
& =\max \{0,3\}=3 \leq r \sigma(x, y) \leq r M(x, y) .
\end{aligned}
$$

case(3): $x=y=1$. We have

$$
\begin{aligned}
H_{\sigma}(T x, T y)=H_{\sigma}(\{0,2\},\{0,2\}) & =\max \{\sigma(0,\{0,2\}), \sigma(2,\{0,2\})\} \\
& =\min \{\sigma(0,2), \sigma(0,0)\}=1 \\
& \leq r \sigma(1,1) \leq r M(1,1) .
\end{aligned}
$$

Hence, for all $x, y \in X$

$$
\left.\psi(r) \sigma(x, T x) \leq \sigma(x, y) \quad \text { implies } \quad H_{\sigma}(T x, T y)\right) \leq r M(x, y) .
$$

Thus, all hypotheses of Theorem 2.4 are satisfied. Then, $T$ has a fixed point which is $u=2$. 
3.2. Application. Generally, a dynamical process consists of a state and a decision space. The state space is the set of the initial state, actions and transition model of the process; the decision space is the set of possible actions that are allowed for the process.

In this section, we assume that $U$ and $V$ are Banach spaces and $W \subset U$ is a state space and $D \subset V$ is a decision space. It is well known that the dynamic programming provides useful tools for mathematical optimization and computer programming as well. In particular, the problem of dynamics related to multistage process reduces the problem of solving the functional equation

$$
q(x)=\sup _{y \in D}\{f(x, y)+G(x, y, q(\tau(x, y)))\}, \quad x \in W,
$$

which further can be reformulated as

$$
q(x)=\sup _{y \in D}\{f(x, y)+G(x, y, q(\tau(x, y)))\}, \quad x \in W,
$$

where $\tau: W \times D \rightarrow W, f: W \times D \rightarrow \mathbb{R}, G: \times D \times \mathbb{R} \rightarrow \mathbb{R}$. Here, we study the existence and uniqueness of the bounded solution of the functional equation (3.1).

Let $B(W)$ denote the set of all bounded real-valued functions on $W$ and, for an arbitrary $h \in B(W)$, define $\|h\|=\sup _{x \in W}|h(x)|$. Clearly, $(B(W),\|\cdot\|)$ is a Banach space. Take the metric-like $\sigma$ defined by

$$
\sigma(h, k)=\sup _{x \in W}|h(x)|+\sup _{x \in W}|k(x)|
$$

for all $h, k \in B(W)$. Clearly, $(B(W), \sigma)$ is a complete metric-like space. We also define $T: B(W) \rightarrow B(W)$ by

$$
T(h)(x)=\sup _{y \in D}\{f(x, y)+G(x, y, h(\tau(x, y)))\}, \quad x \in W,
$$

for all $h \in B(W)$ and $x \in W$. Obviously, if the functions $f$ and $G$ are bounded, then $T$ is well-defined. Finally, let

$$
M(x, y):=\max \left\{\sigma(x, y), \sigma(x, T x), \sigma(y, T y), \frac{1}{4}\{\sigma(x, T y)+\sigma(y, T x)\}\right\} .
$$

We will prove the following theorem.

TheOREM 3.3. Assume that there exists $0 \leq r<1$ such that

$$
\left.\sup _{y \in D} \| f(x, y)+G(x, y, h(\tau(x, y)))\right\} \| \leq r \sup _{x \in W}|h(x)|,
$$

where $x \in W, y \in D, T: B(W) \rightarrow B(W)$ is given by (3.2) and the functions $G: W \times D \times \mathbb{R} \rightarrow \mathbb{R}$ and $f: W \times D \rightarrow \mathbb{R}$ are bounded. Then the functional equation (3.1) has a unique bounded solution. 
Proof. From (3.3), for all $x \in W, y \in D, h \in B(W)$ and $k \in B(W)$, we have

$$
\begin{aligned}
\sigma(T(h), T(k))= & \sup _{y \in D}|f(x, y)+G(x, y, h(\tau(x, y)))| \\
& +\sup _{y \in D}|f(x, y)+G(x, y, k(\tau(x, y)))| \\
\leq & r \sup _{x \in W}|h(x)|+r \sup _{x \in W}|k(x)| \\
\leq & r \sigma(h, k) \leq r M(h, k) .
\end{aligned}
$$

The mapping $T$ has a unique fixed point, that is, the functional equation (3.1) has a unique bounded solution.

\section{ACKNOWLEDGEMENTS.}

The authors gratefully acknowledge the support from King Abdulaziz City for Sciences and Technology (KACST), Kingdom of Saudi Arabia, Project Number (SG: 36-39).

\section{REFERENCES}

[1] C. T. Aage and J. N. Salunke, The results on fixed points in dislocated and dislocated quasi-metric space, Appl. Math. Sci. (Ruse) 2 (2008), 2941-2948.

[2] M. Abbas, B. Ali and C. Vetro, A Suzuki type fixed point theorem for a generalized multivalued mapping on partial Hausdorff metric spaces, Topology Appl. 160 (2013), $553-563$.

[3] A. Amini-Harandi, Metric-like spaces, partial metric spaces and fixed points, Fixed Point Theory Appl. 2012, 2012:204, 10pp.

[4] H. Aydi, M. Abbas and C. Vetro, Partial Hausdorff metric and Nadler's fixed point theorem on partial metric spaces, Topology Appl. 159 (2012), 3234-3242.

[5] H. Aydi, M. Abbas and C. Vetro, Common fixed points for multivalued generalized contractions on partial metric spaces, Rev. R. Acad. Cienc. Exactas Fs. Nat. Ser. A Math. RACSAM 108 (2014), 483-501.

[6] H. Aydi and A. Felhi, On best proximity points for various $\alpha$-proximal contractions on metric-like spaces, J. Nonlinear Sci. Appl. 9 (2016), 5202-5218.

[7] H. Aydi, A. Felhi, E. Karapinar and S. Sahmim, Hausdorff metric-like, generalized Nadler's fixed point theorem on metric-like spaces and application, Miskolc Math. Notes, in press.

[8] H. Aydi, A. Felhi and S. Sahmim, Fixed points of multivalued nonself almost contractions in metric-like spaces, Math. Sci. (Springer) 9 (2015), 103-108.

[9] H. Aydi and E. Karapinar, Fixed point results for generalized $\alpha-\psi$-contractions in metric-like spaces and applications, Electron. J. Differential Equations 2015, No. 133, $15 \mathrm{pp}$.

[10] R. D. Daheriya, R. Jain and M. Ughade, Some fixed point theorem for expansive type mapping in dislocated metric space, ISRN Math. Anal. 2012, Art. ID 376832, 5 pp.

[11] R. George, R. Rajagopalan and S. Vinayagam Cyclic contractions and fixed points in dislocated metric spaces, Int. J. Math. Anal. (Ruse) 7 (2013), 403-411.

[12] A. Isufati, Fixed point theorems in dislocated quasi-metric space, Appl. Math. Sci. (ruse) 4 (2010), 217-233.

[13] E. Karapinar and P. Salimi, Dislocated metric space to metric spaces with some fixed point theorems, Fixed Point Theory Appl. 2013 (2013). 
[14] P. S. Kumari, W. Kumar and I. R. Sarma, Common fixed point theorems on weakly compatible maps on dislocated metric spaces, Math. Sci. (Springer) 6 (2012), Art. 71, $5 \mathrm{pp}$.

[15] P. S. Kumari Some fixed point theorems in generalized dislocated metric spaces, Math. Theory Model. 1 (2011), 4, 16-22.

[16] S. G. Matthews, Partial metric topology, in Proceedings of the 8th Summer Conference on General Topology and Applications. Annals of the New York Academy of Sciences 728, 1994, 183-197

[17] S. B. Nadler, Jr., Multi-valued contraction mappings, Pacific J. Math. 30 (1969), 475488.

[18] I. R. Sarma and P. S. Kumari, On dislocated metric spaces, Int. J. Math. Arch. 3 (2012), 1, 72-77.

[19] R. Shrivastava, Z. K. Ansari and M. Sharma, Some results on fixed points in dislocated and dislocated quasi-metric spaces, J. Adv. Stud. Topol. 3 (2012), 25-31.

[20] M. Shrivastava, K. Qureshi and A. D. Singh, A fixed point theorem for continuous mapping in dislocated quasi-metric spaces, Int. J. Theor. Appl. Sci. 4 (2012), 1, 39-40.

[21] K. Zoto, E. Hoxha and A. Isufati Some new results in dislocated and dislocated quasimetric spaces, Appl. Math. Sci. (Ruse) 6 (2012), 3519-3526.

\author{
H. Aydi \\ Department of Mathematics \\ University of Dammam \\ College of Education of Jubail \\ 31961 Jubail \\ Saudi Arabia \\ \& \\ Department of Medical Research \\ China Medical University Hospital \\ China Medical University \\ Taichung, Taiwan \\ E-mail: hmaydi@uod.edu.sa
}

A. Felhi

Department of Mathematics and Statistics

King Faisal University

College of Sciences

31928 Al Ahsaa

Saudi Arabia

E-mail: afelhi@kfu.edu.sa

S. Sahmim

Department of Mathematics and Statistics

King Faisal University

College of Sciences

31928 Al Ahsaa

Saudi Arabia

E-mail: ssahmim@kfu.edu.sa

Received: 16.3.2016.

Revised: 2.12.2016. 